\title{
PREVALANCE, RISK FACTORS AND OUTCOMES OF VENTILATOR ASSOCIATED PNEUMONIA IN CHILDREN ADMITTED IN PEDIATRIC INTENSIVE CARE UNIT IN BIKANER, NORTHWETERN INDIA
}

\section{Gajanand Singh Tanwar*, Priya Tanwar, Heena Gahlot, Renu Agrawal; Department of Pediatric Medicine, S.P. Medical College, Bikaner}

\section{Background}

Ventilator-associated pneumonia (VAP) is defined as nosocomial pneumonia in mechanically ventilated patients that was not present at the time of intubation, with onset after 48 hours of PICU stay. The epidemiology and outcomes of ventilator associated pneumonia are well described in adults. Although children have different anatomy and physiology, have different underlying illnesses, and undergo different surgical procedures from adults, few data exist for pediatric patients, particularly with respect to risk factors and outcomes. This prospective study was executed to determine the prevalence, the risk factors and outcomes associated with VAP in children admitted in pediatric intensive care unit (PICU) in PBM children hospital.

\section{Meterials and Methods}

This prospective cohort study enrolled all children $(\leq 15$ years aged) who were admitted to the PICU from January 2017 to December 2017, except those who died within 24 hours. The primary outcome measured was the development of VAP. For diagnosing VAP, the patient was required to have received at least 48 hours of mechanical ventilation and develop new and persistent radiographic evidence of focal infiltrates 48 hours or more after the initiation of mechanical ventilation.
In addition, patients had to have 2 of the following: fever $\geq 38^{\circ} \mathrm{C}$, leukocytosis (white blood cell $\geq 12000 / \mathrm{mm}$ ), and purulent sputum ( $\geq 25$ white blood cells/high poweredfield on tracheal aspirate Gram stain). Associated organisms were designated as those organisms recovered from tracheal aspirates or bronchoalveolar lavage from patients with ventilator associated pneumonia. Secondary outcomes were death and length of stay in PICU. Multiple logistic regression analysis was performed to determine independent predictors for VAP.

\section{Results}

There were 124 episodes of VAP in 334 mechanically ventilated children. There was a slight predominance of male children. The mean age was $4.47 \pm 4.90$ years (median age: 3.94 years). The mean VAP rate was 11.6/1000 ventilator days. Patients with VAP had been in the PICU for a mean of 8.9 days before the development of VAP. There was a preponderance of Gram-negative organisms, particularly Pseudomonas aeruginosa and Klebsiella pneumoniae. Among Gram positive organisms Staphyococcus aureus and Strptococcus pneumoniae were predominant. The mean and median PRISM scores were $8.48 \pm 6.13$ and 7.00, respectively. Lung diseases (35\%), congenital heart disease (29\%), neurological diseases and genetic syndrome (25\%) were common predictors to mechanical ventilation, whereas burns and congenital immunodeficiency were less common. Ventilator-associated pneumonia was associated with the following procedures: reintubation, cardio-pulmonary resuscitation, dialysis, blood component transfusion, transport out of the PICU, tracheostomy, the presence of a central line and multiple central venous catheters. Total parental nutrition, steroids, and histamine type 2 receptor blockers were common drugs that associated with VAP. By multivariate logistic regression analysis, genetic syndrome $(\mathrm{OR}=2.37 ; 95 \% \mathrm{CI}: 1.01-5.46)$, reintubation $(\mathrm{OR}=2.71 ; 95 \%$ CI: 1.18-6.21), and transport out of the PICU $(\mathrm{OR}=8.90$; 95\% CI: 3.82-20.74) independently predicted VAP.

\section{Conclusion}

We performed a prospective cohort study to determine rates of, risk factors for, and outcomes associated with VAP in PICU. We found a relatively high rate of VAP and identified independent predictors of ventilator-associated pneumonia, including genetic syndrome, reintubation, and transport out of the PICU. Patients with ventilator associated pneumonia had longer PICU stay, and the difference in mortality approached statistical significance. Studies of interventions to decrease ventilator-associated pneumonia are needed in PICU patients. 